\title{
Ontology Model for Situation Awareness of City Tunnel Traffic
}

\author{
Lin $\mathrm{Li}^{1}$,Weiguo $\mathrm{Wu}^{2}$ \\ ${ }^{1}$ School of Computer Science and Technology, \\ ${ }^{2}$ School of Transportation, \\ Wuhan University of Technology, \\ Wuhan, China \\ cathylilin@whut.edu.cn mailjt@163.com
}

\author{
Nian $\mathrm{Liu}^{3}$ \\ ${ }^{3}$ School of Computer Science and Technology, Wuhan \\ University of Technology, \\ Wuhan, China \\ nianlau@sina.cn
}

\begin{abstract}
Networking of city tunnel monitoring systems is an application instance of Internet of Things. Situation awareness of city tunnel traffic is a method of analyzing the traffic safety of city tunnel. This paper studies the ontology model for monitoring tunnel traffic situation. The model reflects the basic concepts and semantic meanings of an integrated traffic network. The situation of the integrated traffic network can be described in standard formats. The use and management of the integrated traffic network is improved. Therefore this model can improve the effectiveness and efficiency of intelligent transportation service.
\end{abstract}

Keywords: city tunnel traffic; ontology model; situation

\section{INTRODUCTION}

With acceleration of urbanization, city long tunnels and the super long tunnels have been constructed. According to statistics, the number of already built or under construction tunnels were above several thousands. The city tunnel has accumulated a large amount of monitoring data through the establishment of monitoring system, including environmental data, traffic data, and video data. In order to obtain valuable information about these monitoring data, data sharing between multiple city tunnel monitoring systems can be realized, and provide support for data mining. Then we can use data mining technology to discover the potential of knowledge, and realize traffic situation awareness to manage the city traffic efficiently. In addition, it will provide services for public safety, traffic control, and urban management, thus the concept of Internet of Things technology is further put into application.

This paper will analyze the data characteristics of city tunnel traffic using ontology method to establish ontology model for traffic network situation awareness ${ }^{[2]}$. The model reflects the basic concepts and semantic meanings of an integrated traffic network situation. We can describe the situation of the integrated traffic network, and support the use and management of the integrated traffic network to improve the service efficiency by using the model ${ }^{[3-4]}$.

\section{THE TUNNEL TRAFFIC SITUATION ONTOLOGY MODEL}

We adopt OWL that is based on XML by increasing modeling primitives to describe class, properties, instances of class and their relationship. We provide type definitions and attribute descriptions for features, thus establish the tunnel traffic situation ontology model.

From the main data source, traffic area knowledge, management and organization, traffic resource ontology set are defined into three areas of sub-ontology. In the city tunnel monitoring system, monitoring points will have a wide variety of real-time continuous produced monitoring data. It can be divided into monitoring sensor data and control sensing data.

\section{A. Monitoring ontology}

It provides concepts and relationship that are related with monitoring information, including tunnel environmental data: CO/VI, wind speed/direction, light intensity /illumination, temperature/humidity values, the water level values, etc. The tunnel traffic data includes traffic flow, speed, lane occupancy rate, etc. Class hierarchy is shown in Figure 1.

The hierarchy of monitoring portion includes discrete monitoring and analogue type monitoring. Discrete monitoring includes lighting monitoring data, pump monitoring data, blower monitoring data, and lane lamp monitoring data. The analogue type monitoring includes visibility monitoring sensor, wind speed monitoring sensor, co monitoring sensor, light intensity monitoring sensor, pump monitoring sensor.

The monitoring equipment can monitor the tunnel environment and monitoring device's own 


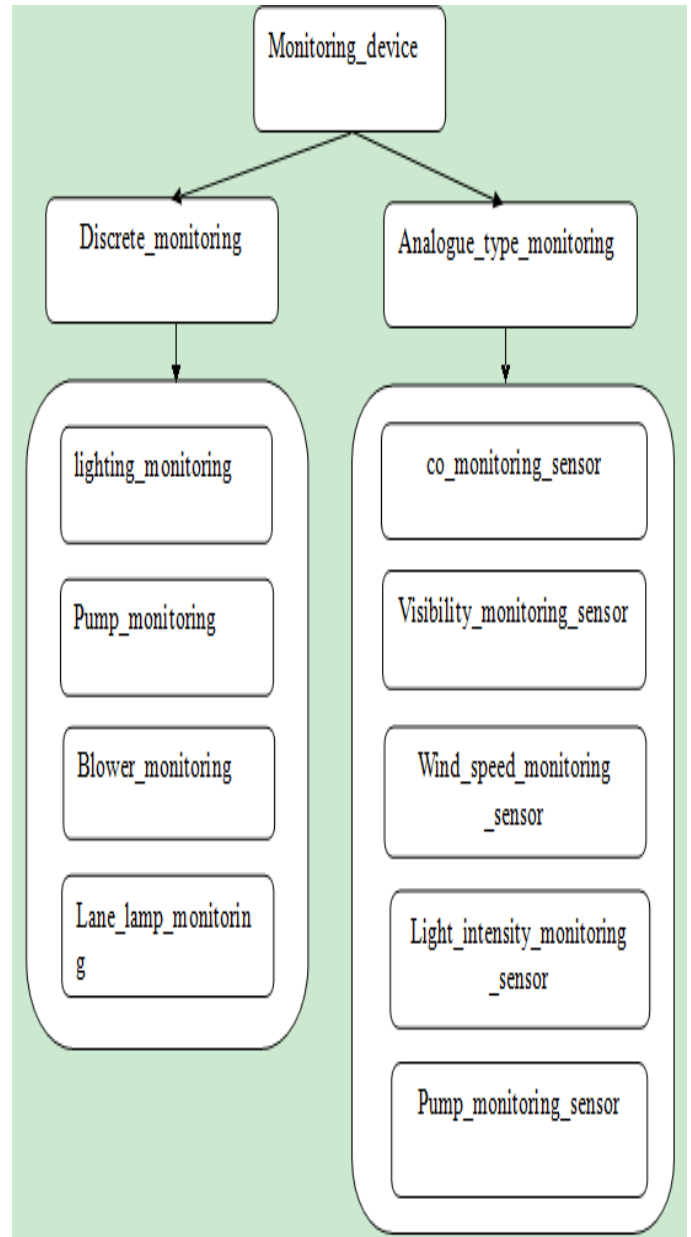

Figure1 monitoring ontology hierarchy

status. The analogue monitoring data reflects the tunnel visibility, wind speed, co concentration, light intensity,

According to the tunnel environment, the operating device will change control state, thus affecting the tunnel environment. Multimode control data reflect the situation of blower control and lane lamp control. When wind speed is relatively low and affects co concentration conditions, we can turn on the blower control. The minimum speed of blower control is $5 \mathrm{~m} / \mathrm{s}$ and the maximum speed of blower control is $12 \mathrm{~m} / \mathrm{s}$. Light in the tunnel is more dark than light outside of the tunnel, so in order to facilitate traffic we need turn on the lane lamp lights. The number of opened lane lamp is relevant to light in the tunnel. At least the number of opened lane lamp is 12, and most the number of opened lane lamp is 48.

\section{Traffic ontology}

The traffic ontology provides concepts and relationships that are associated with traffic information. There are two categories, i.e., static ontology and event ontology. Static ontology describes inherent and physical concept, such as vehicles, traffic lights, equipment and the water level in the tank. Tunnel visibility can not be less than $6 \mathrm{~m}$, and the wind normal value is $8 \mathrm{~m} / \mathrm{s}$. co concentration shall not be higher than 250.00ppm. The tunnel light intensity is $70.00 \mathrm{~cd} / \mathrm{m}$ and the water level in the tank is $15 \mathrm{~cm}$.

\section{B. Control ontology}

It provides the current state of operating device: blower control, lighting control, pump control, lane lamp control. Class hierarchy is as shown in Figure 2.Single state control is a single control, such as lighting control has only a state .Multiple state controls are a combination control state of several control states, such as blower control is a controlled combination of the forward switch and reverse switch.

The hierarchy of operating device includes single state control and multiple state controls. Single state control includes lighting control, pump control. Multiple state controls include blower control, lane lamp control.

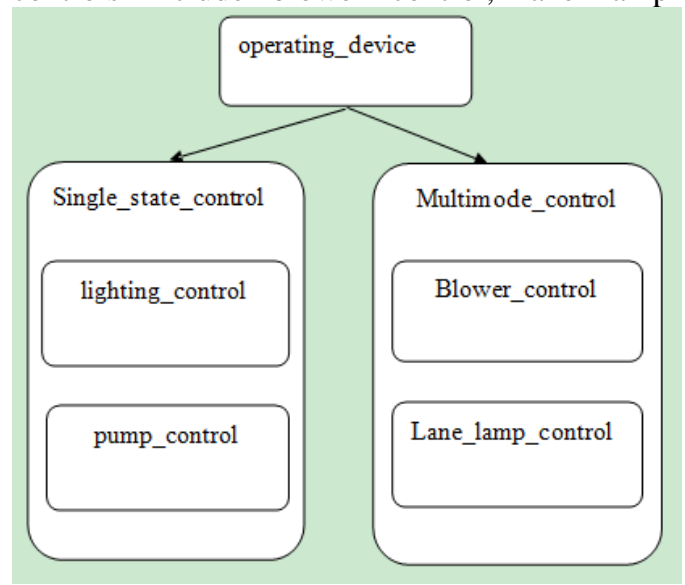

Figure 2 control ontology hierarchy

sensors. The event ontology describes temporary and action concept, such as traffic jams, accidents, fire, blast, and so forth.

\section{Manager ontology}

To realize cooperative work of various departments, we need define the mutual relationship and data access of the various departments. Traffic organization ontology provides knowledge representation for collaboration between organizations, including traffic management centers, traffic information service providers, emergency management departments, infrastructure management departments, cargo transportation services providers, government law enforcement departments, and so forth.

\section{THE REALIZATION AND USE OF MODEL}

The development tool of ontology modeling is Protege. Protege Software, which was developed by Stanford University, is an ontology editor and knowledge acquisition software based on the Java language developed. It is not only an ontology development tool, 
but also a knowledge-based editor, belonging to the open-source software. Protege provides ontology concept class, relationships, properties and the construction of instances, and shields the specific ontology language.

The tool of ontology modeling is Protege 4.1 version in this paper. Class hierarchy, object properties and data type properties of monitoring device ontology are shown below

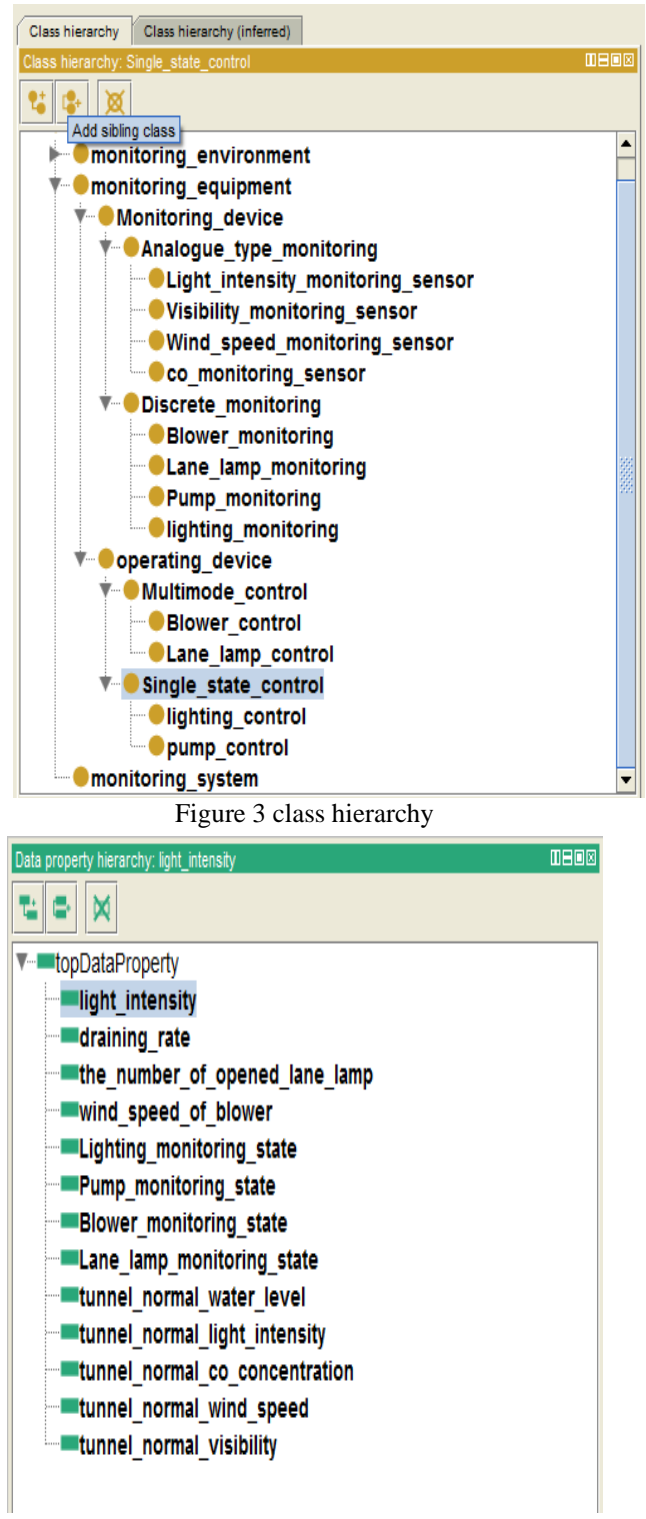

Figure 4 data type properties

From Fig 3 and Fig.4, we can see a good concept hierarchy and the support of logical reasoning of domain ontology, which fully discovers the semantic relationships that implied in the data internally, and provides a rich data base for the query. Semantic query system, which is based on it, excludes irrelevant query results that query information can not articulate, and effectively improve the quality and efficiency of the field of information query.

\section{CONCLUSION}

Expansion of China's urbanization promotes the rapid development of the city tunnel and the rapid expansion of monitoring data, and decision service demand provided by urban information management is also increasing. This paper studies and designs four sub-ontologies, i.e., monitoring, traffic situation awareness operating, traffic and management, which provides new ideas and methods for the city intelligent traffic decision support system.

\section{REFERENCES}

[1] Lakkaraju K, Yurcik W, Lee A J. NVisionIP: NetFlow visualizations of system state for security situational awareness[C]. In: Proceedings of the 2004 ACM Workshop on Visualization and Data Mining for Computer Security,Washington,DC,2004: 65-72.

[2] Endsley M.R., Sollenberger R., and Stein E. Situation awareness: A comparison of measures[C]. In Proceedings of the Human Performance, Situation Awareness and Automation: User-Centered Design for the New Millennium, 2000.

[3] Endsley M.R., Rodgers M.D. Distribution of attention, situation awareness, and workload in a passive air traffic control task: Implications for operational errors and automation[J]. Air Traffic Control Quarterly,1998, 6(1):21-44.

[4] Jones D.G., Bolstad C.A., Riley J.M. Situation awareness requirements for the future objective force[C]. Proceedings of the ARL Collaborative Technology Alliances Conference, 2003.

[5] Dean Allemang,James Hendler .Semantic Web for the Working Ontologist[C].Morgan Kaufmann,2011. 